\title{
A linear model of the effects of residence time distribution on mixing pattern in a ventilated airspace
}

\author{
Chung-Min Liao*, Huang-Min Liang \\ Department of Bioenvironmental Systems Engineering, National Taiwan University, Taipei, 10617, Taiwan, ROC
}

Received 7 January 2002; accepted 10 January 2002

\begin{abstract}
The ability of a simple linear response model is evaluated to explain the mixing efficiencies in an incomplete mixing ventilated airspace. Data interpretation and mean residence time calculation for a specified output concentration profile can also be evaluated. The residence time distribution (RTD) functions take the form of the two-parameter gamma distribution and account for different mixing types such as complete mixing, piston flow (no mixing), incomplete mixing, and various combinations of the above types. In these combinations, the different mixing types simulated by different RTDs conceptually represent airflow regions in series. The mixing efficiency was introduced to characterize the extent or degree of mixing in a ventilation system in that mixing efficiency equals zero for piston flow (no mixing), unity for complete mixing, and a value in between these two extremes for incomplete mixing. An environmental chamber experiment was conducted to generate several output profiles to evaluate the applicability of the model. Carbon dioxide was employed as the tracer gas. Our results show that an overall root-mean-squared error value of $8.64 \pm 5.25 \mathrm{ppm}$ is low, indicating that the combination mixing patterns are generally found to be minimally biased and give better fitting than other simpler mixing patterns. Despite their neglect of molecular diffusion and possible temporal/spatial nonlinearities, these linear response models appear reasonably robust, making them at least as useful to building microenvironment designers in reconsidering the possibilities and consequences of various forms of incomplete mixing related to indoor air quality problems. (C) 2002 Elsevier Science Ltd. All rights reserved.
\end{abstract}

Keywords: Indoor air quality; Mixing; Residence time distribution; Ventilation

\section{Introduction}

Knowledge of the mixing patterns in a ventilated airspace is valuable at both the design stage and during operation to determine the effective mixed proportion of the air volumes and existing contaminants and to assess the values of different operational procedures as a means of optimizing process performance in indoor air quality control problems. Barber and Ogilvie [1] suggested that departure from complete mixing might be caused by the formation of multiple flow regions within the airspace or by short-circuiting of supply air to exhaust outlet. A work presented by Chen et al. [2] regarding the methods to measure dust production and deposition rates in buildings indicated that the assumption of complete mixing was not valid during tests. Their

\footnotetext{
${ }^{*}$ Corresponding author. Tel.: +886-2-2363-4512; fax: $+886-2-2362-$ 6433.

E-mail address: cmliao@ccms.ntu.edu.tw (C.-M. Liao).
}

experiments showed that tanks-in-series flow, i.e., different flow regions behaving as a number of mixed tanks connected in series dominated the overall mixing process within the ventilated airspace. Their work also suggested that a more complicated multi-zone mixing model might be needed to account for incomplete mixing to better understand the behavior of dust in local transport mechanisms.

Levenspiel [3] indicated that mixing in a continuous flow system could range from piston flow (plug flow or no mixing) as a lower limit, to complete mixing as an upper limit. Both complete mixing and piston flow represent the limiting cases that seldom occur in ventilation systems. It is most probable that the type of mixing encountered in ventilation systems, in general, lies somewhere between these two extremes. We call this type as incomplete mixing or partial mixing.

The most widely used analytical model for simulating the airflow patterns and convection-diffusion distribution of a contaminant in building environment is the dispersive 


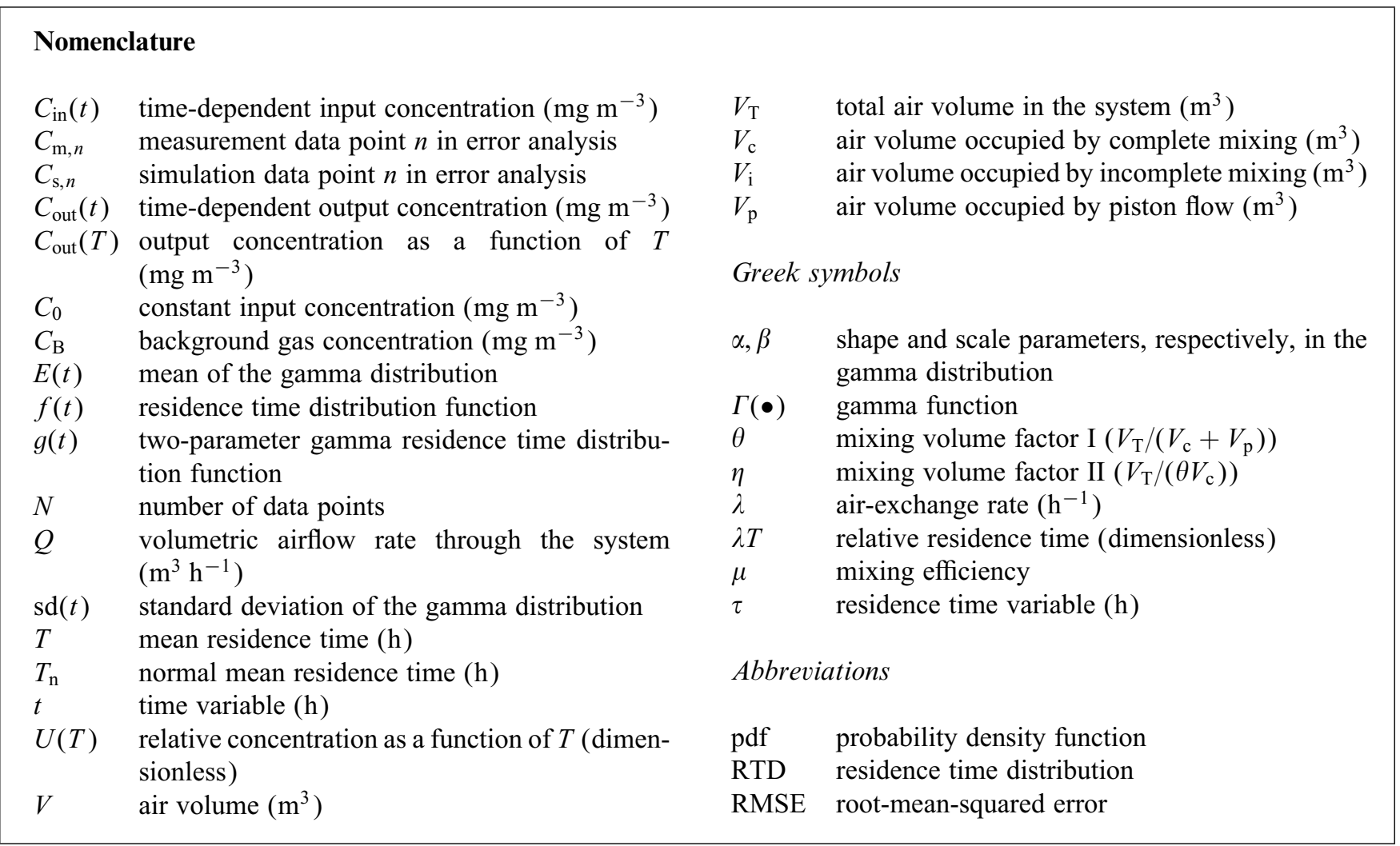

model that utilizes the dispersivity concept in a distributed scheme [4-8]. Reliable estimates of the dispersivity may be scale-dependent, however, are very difficult to obtain. The dispersive model is also characterized by other limitations. Liu et al. [7] and Hoff and Bundy [9] pointed that the theoretical foundation for the dispersive model in its multiple airflow regions is not sound for systems other than air jet with turbulent or laminar flow.

The main parameter describing the temporal change in a continuous system is the mean residence time (or mean age) of air in the ventilation system [10]. For time-invariant systems, the mean age $T$ is defined as [10] $T=\int_{0}^{\infty} t f(t) \mathrm{d} t$, where $f(t)$ is the residence time distribution (RTD) function which describes the exit time distribution of fluid element (air) entering the system at a given time $t=0$. The RTD approach can also be used to describe aerodynamic transport in a ventilated airspace. The methodology is general in the sense that the heterogeneity in transport properties may also be incorporated using various RTDs of specific interest.

Mathematically, $f(t)$ is the probability density function, for the time $t$. Etheridge and Sandberg [11], Freeman et al. [12], Skarret and Mathisen [13], Grot and Lagus [14] and among others pointed out that $f(t)$ could be obtained by a tracer gas (e.g., $\mathrm{SF}_{6}$ ) experiment or by measuring the existing gaseous pollutant concentrations (e.g., $\mathrm{CO}_{2}$, $\mathrm{NH}_{3}$ ) presented in the ventilated airspace. A parsimonious representation of $f(t)$ can be obtained by a simple unimodel and continuous mathematical function. A convenient and flexible mathematical function is the gamma density function. The gamma distribution is a distribution of Pearson's Type III in statistics. The unique feature of the gamma distribution is that one end of the distribution is bound to a fixed value, whereas the other end is distributed over a large scale of the variate. The overall shape of the gamma distribution is not balanced as a normal distribution. The other reason to utilize the gamma distribution is that this approach may reduce the mathematical terms in the analytical solution.

Three most common mixing patterns appearing in a continuous flow system are the complete mixing, piston flow, and the complete-piston flow that simulates the combination of complete mixing and piston flow [10]. The new mixing patterns discussed in this study are the completeincomplete-piston flow, complete-incomplete mixing, incomplete-piston flow, and incomplete mixing patterns. It is obvious that incomplete mixing alone or in combination with the other mixing types is more likely to occur in a general ventilation system than piston flow alone, complete mixing alone, or the combination of complete mixing-piston flow.

A logical first step in this assessment is an evaluation of ability of a simple linear response function model to account for different types of mixing in general ventilation systems such as piston flow (no mixing), incomplete mixing, complete mixing and their combinations which represent the simultaneous existence of the three or any two of the mixing types in the system. The proposed linear response model is free from the limitations of the dispersive model (i.e., the 
dispersivity problem) that makes them an effective tool for investigating indoor air quality problems. The linear response model is particularly useful for investigating systems that lack detailed ventilation data, i.e., systems with unknown distribution of parameters. Considering that ventilation systems in many commercial buildings and/or animal housing are characterized by little or no ventilation data, analysis of tracer data by the linear response model may be the only available approach for solving air-supply problems.

The significance of this work has two effects: (1) this study will alert designers of building environment to the possibilities and consequences of various forms of incomplete mixing, and (2) the model can be used to quantify mixing characteristics to replace the complete mixing assumption in ventilation design calculation. Because of the inherent difficulties in field measurements, an environmental chamber experiment was conducted to evaluate the ability of the model under controlled conditions.

\section{Materials and methods}

\subsection{Environmental chamber experiments}

The test facilities used in the experimental studies were composed of an environmental chamber, an air delivery system, a carbon dioxide gas generating system and a carbon dioxide gas sampling system. The ambient temperature during experiment was $31 \pm 0.6^{\circ} \mathrm{C}$. The relative humidity ranged between $70 \%$ and $80 \%$. We employed carbon dioxide as the tracer gas.

Fig. 1 gives the dimensions and general outline of the environmental chamber. The dimensions were $1.54 \times 0.92 \times$ $0.95 \mathrm{~m}^{3}$ for a volume of $1.35 \mathrm{~m}^{3}$. The dimensions were such as to simulate one slot-ventilated enclosure with a negative pressure ventilation system. The chamber, fabricated from one Plexiglas and three sandwich-type boards.

Air entered the chamber through a long slot inlet measuring $92 \times 4.5 \mathrm{~cm}^{2}$. A $30 \mathrm{~cm}$ diameter, 6-blade propeller fan was used to exhaust air from the chamber. The exhaust location was $80 \mathrm{~cm}$ from floor level measured to the center of the outlet. The fan operated at constant speed by a voltage indicator maintaining a constant air velocity. Fan output expressed as $\mathrm{m}^{3} \mathrm{~h}^{-1}$ was measured near the end of a discharged duct. Chamber ventilation rates were determined using the impeller method based on manufacturer specification. Ventilation rates used throughout the experiments were ranged from 850 to $1070 \mathrm{~m}^{3} \mathrm{~h}^{-1}$ to generate various forms of carbon dioxide concentration output profiles.

Gas generating system consisted of a high-pressure cylinder of carbon dioxide, a two-stage pressure regulator, plastic tubing, and three flowmeters (Hsin Chuan M-type, No. HC-A02, Taiwan) (Fig. 1). Discharge from the cylinder of pure carbon dioxide was divided into three for the generating operation. The Hsin Chuan M-type flowmeter had a millimeter scale that could be converted to a volume flow scale for air with aid of calibration charts. Adjustment factors associated with the calibration charts were used to calculate the gas flow rate for gases other than air. According to manufacture information, the adjustment factor is equal to the square root of the specific gravity of air divided by the specific gravity of carbon dioxide. The resulting value is 0.808 . Carbon dioxide was used at a rate of $5001 \mathrm{~h}^{-1}$. In order to investigate the variation of carbon dioxide concentration influenced by the spatial change, the carbon dioxide generating points were deployed as shown in Fig. 1.

Two KRK Model 200-type carbon dioxide meters (Kasahara Chemical Instruments Corp., Japan) were used to measure carbon dioxide concentration. Measuring principle for KRK Model 200-type carbon dioxide meter is based on the potentiometric methods in that carbon dioxide gas passed into the membrane of the electrode system in contacting with $\mathrm{NaHCO}_{3}$ solution results in a change of $\mathrm{pH}$ values. Carbon dioxide concentration could be measured through the potential differences derived from the proportional relations between the change of $\mathrm{pH}$ value and the partial pressure of carbon dioxide gas.

Before the chamber test, two carbon dioxide meters were calibrated with known carbon dioxide concentrations of $0 \%$ and $10 \%$, respectively. Sampling was carried out in two planes, 30.7 and $61.4 \mathrm{~cm}$ from a side wall along the $y$-direction of Fig. 1 and the sampling interval was $10 \mathrm{~s}$. All sampling outputs were recorded on a computer-controlled data acquisition system (Fluke-2280A Data Logger, John Fluke MFG Co., Inc., U.S.A.). Manufacturer specification shows that the carbon dioxide reading to be accurate within 3\% might be expected, indicating that the random error of $\pm 3 \%$ was uniformly distributed to carbon dioxide concentration outputs.

\subsection{Mathematical model}

A simple linear response model is proposed to describe the tracer dynamics between output and input gas concentrations subjected to an appropriate RTD function,

$C_{\text {out }}(t)=\int_{0}^{t} C_{\text {in }}(t-\tau) g(\tau) \mathrm{d} \tau+C_{\mathrm{B}}$

where $C_{\text {out }}(t)$ is the output gas concentration $\left(\mathrm{mg} \mathrm{m}^{-3}\right)$, $C_{\text {in }}(t)$ is the input gas concentration $\left(\mathrm{mg} \mathrm{m}^{-3}\right), g(t)$ is the RTD function, also called the system response function or the weighting function, and $C_{\mathrm{B}}$ is the background gas concentration $\left(\mathrm{mg} \mathrm{m}^{-3}\right)$. In the second term of Eq. (1), $t$ is the calendar time, and $\tau$ is the integration variable that represents the residence time of airflow.

In the case of the constant tracer material input with a constant air-exchange rate $\lambda\left(\mathrm{h}^{-1}\right)$ in a ventilated airspace, the fraction remaining after $t$, is simply $\exp (-\lambda t)$. Thus for 


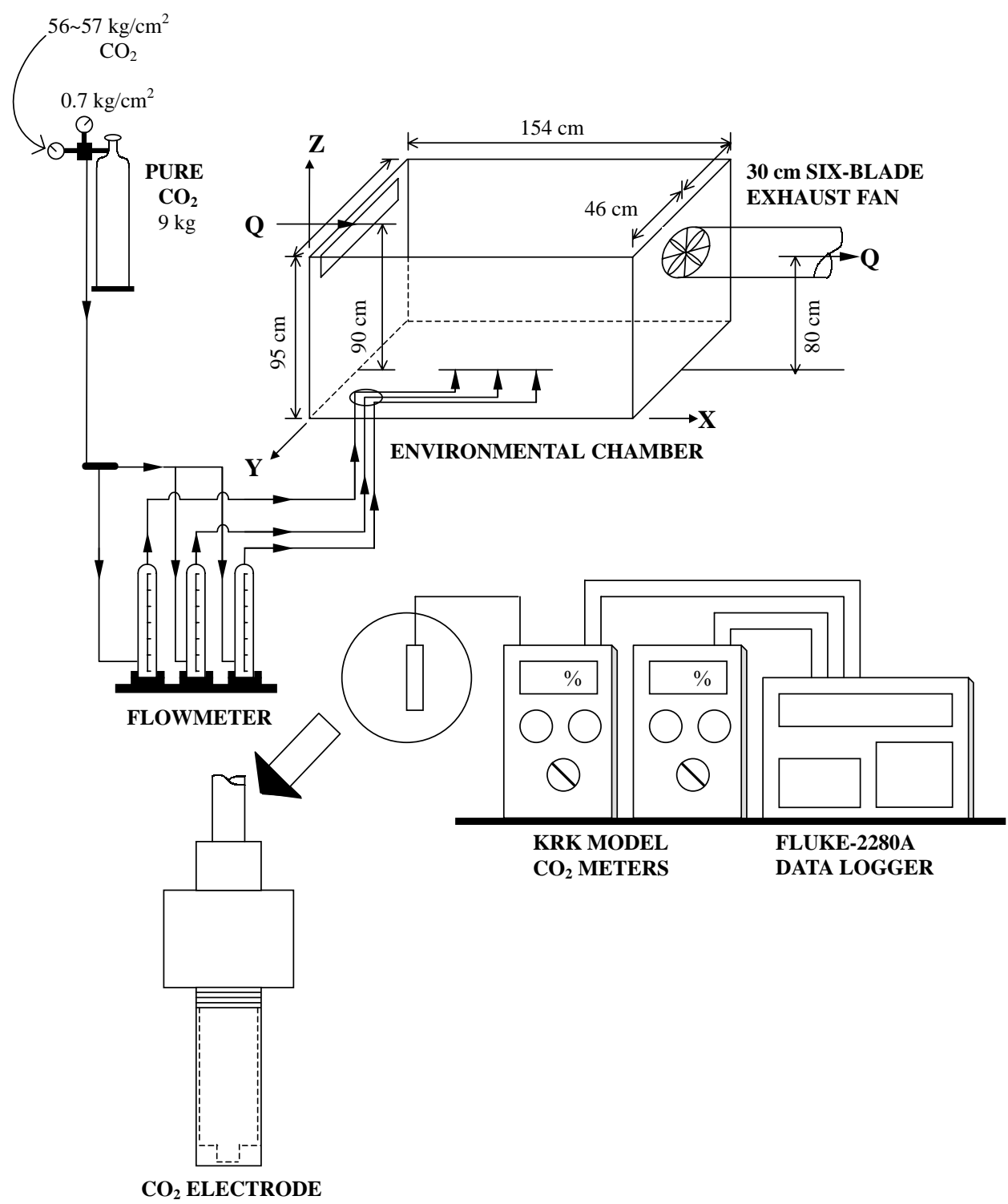

Fig. 1. General outline of the dimensions of environmental chamber and experimental equipments.

total initial input concentration, the linear response model describing the output concentration remaining after $t$ is

$C_{\text {out }}(t)=\int_{0}^{t} C_{\text {in }}(t-\tau) \mathrm{e}^{-\lambda \tau} g(\tau) \mathrm{d} \tau+C_{\mathrm{B}}$.

Our approach is to use the gamma distribution to describe the RTD function in an incomplete mixing of ventilated airspace. Because of the complexity of air mixing, their RTD is not expected to follow a normal distribution. The gamma distribution, thus, has a greater chance to describe the mixing behavior than does the normal distribution. The presented analytical solution demonstrates that it is possible to construct a systematic way of lumping parameters and the incomplete mixing behavior could be analyzed. Buffham and Gibilaro [15] also pointed out that the simplest extension of the tanks-in-series model is the gamma model.

The two-parameter gamma probability density function (pdf) of $t$ may be written as

$g(t) \equiv f(t ; \alpha, \beta)=\frac{1}{\beta^{\alpha} \Gamma(\alpha)} \mathrm{e}^{-t / \beta} t^{\alpha-1} \quad t>0$,

where $\alpha$ (the shape parameter) and $\beta$ (the scale parameter) are positive parameters, the normalization factor $\Gamma(\alpha)=$ $\int_{0}^{\infty} \xi^{\alpha-1} \mathrm{e}^{-\xi} \mathrm{d} \xi$, where $\xi$ is a dummy variable of integration, is the gamma function of mathematical statistics. With the gamma pdf, the mean, $E(t)$ and standard deviation, $\operatorname{sd}(t)$ are: $E(t)=\alpha \beta ; \operatorname{sd}(t)=\alpha^{1 / 2} \beta$, respectively.

If $\alpha \beta$ is held constant, then as $\alpha$ approaches infinity the gamma distribution function approaches the Gaussian distribution, the variance of which decreases as $\alpha$ increases. 
The gamma distribution also satisfies the physical requirement that residence time must be non-negative. The scale parameter $\beta$ serves to determine both the mean and standard deviation of the $t$ 's. The mean and variance of each gamma distribution governs the characteristic properties of the linear response model.

The effect of $\alpha$ and $\beta$ has the following characteristics. For $0<\alpha<1$, much of the air corresponds to very small values of residence time $t$ near 0 ; for $\alpha=1$, an exponential distribution is obtained, for which $t=0$ is still the most likely value. As $\alpha$ increases above 1 the density function becomes increasingly symmetric with small values of $t$ occurring less frequently. Firstly, we are taking the complete-piston flow pattern as an example to get insight into the concept of $\alpha$ and $\beta$.

The occupied air volume relationship among the total volume $\left(V_{\mathrm{T}}\right)$, complete mixing volume $\left(V_{\mathrm{c}}\right)$, and piston flow volume $\left(V_{\mathrm{p}}\right)$ in the complete-piston flow pattern is $V_{\mathrm{T}}=V_{\mathrm{c}}+$ $V_{\mathrm{p}}$. For $\alpha=1$, RTD function in Eq. (3) reduces to

$g(t)=\frac{1}{\beta} \mathrm{e}^{-t / \beta}, \quad t>0$.

Based on Eq. (4) and air volume relations, the complete-piston flow mixing pattern can be mathematically expressed as

$g(t)= \begin{cases}\frac{\eta}{T} \mathrm{e}^{-t \eta / T} & t \geqslant 0, \\ 0, & t<0,\end{cases}$

where

$\beta=\frac{T}{\eta}, \quad \eta=\frac{V_{\mathrm{T}}}{V_{\mathrm{c}}}, \quad T=\frac{V_{\mathrm{T}}}{Q}$.

The complete-piston flow pattern simulates a completely mixed tanks-in-series with a region where airflow moves in piston flow. Therefore, the complete-piston flow is a special case of the two-parameter gamma pdf when $\beta$ is defined by Eq. (6) and $\alpha=1$ and results in the mean and standard deviation of the complete-piston flow are $E(t)=\operatorname{sd}(t)=T / \eta$.

The complete-piston flow combines the complete mixing with the piston flow. For $\eta=1$, the complete-piston flow gives the complete mixing, and for $\eta \rightarrow \infty$, it reduces to the piston flow. Substituting $\eta=1$ or letting $\eta \rightarrow \infty$ in Eq. (6) yields $\beta=T$ for the complete mixing, and $\beta=0$ for the piston flow. The mean is $T$ in both complete mixing and piston flow, and variance is $T^{2}$ for complete mixing and 0 for piston flow.

These values can also be obtained from the mean and variance of the two-parameter gamma pdf. In other words, complete mixing, piston flow, and their combinations (which represent airflow regions in series of the different mixing types) are all accounted for by the two-parameter gamma pdf. For this reason, we propose the general mixing pattern, which takes the functional form of the two-parameter gamma pdf, for simulating mean residence times in ventilation systems, and consequently the additional fitting parameters will give some information about the structure of the system.

The air volume relations of a general mixing pattern of a ventilated airspace in a time-invariant system is

$V_{\mathrm{T}}=V_{\mathrm{c}}+V_{\mathrm{p}}+V_{\mathrm{i}}$

where $V_{\mathrm{i}}$ is the air volume of incomplete mixing region. In a time-varying system, Eq. (7) has the form as $V_{\mathrm{T}}(t)=$ $V_{\mathrm{c}}(t)+V_{\mathrm{p}}(t)+V_{\mathrm{i}}(t)$. Based on Eqs. (6) and (7), after some mathematical manipulation, the shape parameter $\alpha$ and scale parameter $\beta$ can be obtained as

$\alpha=\left\{\begin{array}{l}\frac{V_{\mathrm{T}}}{V_{\mathrm{p}}+V_{\mathrm{c}}+\mu V_{\mathrm{i}}}=\frac{1}{1 / \theta+\mu(1-1 / \theta)}, \quad \mu \geqslant 0.5, \\ \frac{V_{\mathrm{T}}}{V_{\mathrm{p}}+V_{\mathrm{c}}+(1-\mu) V_{\mathrm{i}}}=\frac{1}{1 / \theta+(1-\mu)(1-1 / \theta)}, \quad \mu \leqslant 0.5,\end{array}\right.$

$\beta=\frac{T}{\alpha\left(V_{\mathrm{T}} /\left(V_{\mathrm{c}}+\mu V_{\mathrm{i}}\right)\right)}=\frac{T}{\alpha}\left(\frac{1}{\eta \theta}+\mu-\frac{\mu}{\theta}\right)$

in which

$\theta=\frac{V_{\mathrm{T}}}{V_{\mathrm{c}}+V_{\mathrm{p}}}$

and

$\eta=\frac{V_{\mathrm{T}}}{\theta V_{\mathrm{c}}}$,

where $\mu$ is the mixing efficiency describing the portion of ventilation airflow that has mixed with room air, and $\theta$ and $\eta$ may be referred to as the mixing volume factor I and II, respectively. The detailed deviation of $\alpha$ and $\beta$ is given in Appendix A.

Substituting Eq. (3) into Eq. (2) for a constant $C_{\text {in }}=C_{0}$ gives an output and input relations as a function of $T$ as,

$U(T) \equiv \frac{C_{\text {out }}(T)}{C_{0}}=\frac{1}{(\lambda \beta+1)^{\alpha}}+\frac{C_{\mathrm{B}}}{C_{0}}$,

where $U(T)$ is defined as a relative concentration. Substituting the parameters of $\alpha$ and $\beta$ of any of the mixing patterns into Eq. (12) gives the $U(T)$ of that particular mixing type. In case of the combination mixing patterns of completeincomplete-piston flow,

$U(T)=\frac{1}{[(\lambda T / \alpha)((1 / \eta \theta)+\mu-\mu / \theta)+1]^{\alpha}}+\frac{C_{\mathrm{B}}}{C_{0}}$.

Table 1 summarizes the expressions of the RTD functions and the $U(T)$ s for the several forms of mixing patterns. The first three cases in Table 1 correspond to the mixing patterns of complete-piston flow, complete mixing, and piston flow, respectively. The mixing patterns corresponding to cases 4,5 , and 6 are designated as the incomplete-piston flow, complete-incomplete mixing, and incomplete mixing, respectively. Case 7 represents the combination mixing patterns of complete-incomplete-piston flow. These seven mixing patterns can give other patterns, as special cases, by changing the types of mixing or mixing efficiency. 
Table 1

The residence time distribution (RTD) functions and relative concentration $U(T)$ for different mixing patterns as background gas concentration is negligible

\begin{tabular}{|c|c|c|}
\hline Mixing pattern & RTD function: $g(t)$ & $U(T)=C_{\text {out }} / C_{0}$ \\
\hline 1. Complete-piston & $\frac{\eta}{T} \exp (-t \eta / T)$ & {$\left[\frac{\lambda T}{\eta}+1\right]^{-1}$} \\
\hline 2. Complete mixing & $\frac{1}{T} \exp (-t / T)$ & {$[\lambda T+1]^{-1}$} \\
\hline 3. Piston flow & $\delta(t-T)$ & $\exp (-\lambda T)$ \\
\hline 4. Incomplete-piston & $\frac{t^{\alpha-1}}{\left[\frac{T}{\alpha}\left(\mu-\frac{\mu}{\theta}\right)\right]^{\alpha} \Gamma(\alpha)} \exp \left\{\frac{-t}{\frac{T}{\alpha}\left(\mu-\frac{\mu}{\theta}\right)}\right\}$ & {$\left[\frac{\lambda T}{\alpha}\left(\mu-\frac{\mu}{\theta}\right)+1\right]^{-\alpha}$} \\
\hline 5. Complete-incomplete & $\frac{t^{\alpha-1}}{\left[\frac{T}{\alpha}\left(\frac{1}{\theta}+\mu-\frac{\mu}{\theta}\right)\right]^{\alpha} \Gamma(\alpha)} \exp \left\{\frac{-t}{\frac{T}{\alpha}\left(\frac{1}{\theta}+\mu-\frac{\mu}{\theta}\right)}\right\}$ & {$\left[\frac{\lambda T}{\alpha}\left(\frac{1}{\theta}+\mu-\frac{\mu}{\theta}\right)+1\right]$} \\
\hline 6. Incomplete mixing & $\frac{t^{\alpha-1}}{\left[\frac{T \mu}{\alpha}\right]^{\alpha} \Gamma(\alpha)} \exp \left\{\frac{-t}{\frac{T \mu}{\alpha}}\right\}$ & {$\left[\frac{\lambda T \mu}{\alpha}+1\right]^{-\alpha}$} \\
\hline 7. Complete-incomplete-piston & $\frac{t^{\alpha-1}}{\left[\frac{T}{\alpha}\left(\frac{1}{\eta \theta}+\mu-\frac{\mu}{\theta}\right)\right]^{\alpha} \Gamma(\alpha)} \exp \left\{\frac{-t}{\frac{T}{\alpha}\left(\frac{1}{\eta \theta}+\mu-\frac{\mu}{\theta}\right)}\right.$ & {$\left[\frac{\lambda T}{\alpha}\left(\frac{1}{\eta \theta}+\mu-\frac{\mu}{\theta}\right)+1\right]^{-0}$} \\
\hline
\end{tabular}

Cholette and Cloutier [16] and Jennings and Armstrong [17] pointed out that incomplete mixing describes the type of mixing between piston flow and complete mixing in that the mixing efficiency is introduced and defined. The approaches presented by Cholette and Cloutier [16] and Jennings and Armstrong [17] for characterizing the overall decay rate when mixing is not complete is to fit an expression of the type in terms of measurement data as $C(t) \approx$ $C(0) \exp (-Q / V \mu t)$, in which $C(t)$ is time-dependent tracer concentration, $C(0)$ is the initial condition of tracer concentration, $Q / V=T^{-1}$ is the inverse mean residence time of ventilation system; $Q$ is the ventilation rate, and $V$ is the air volume.

Incomplete mixing can range from near complete mixing to near piston flow, but never reaches these extremes. We introduce the concept of mixing efficiency to specify the exact location of incomplete mixing in the range between piston and complete mixing. In general ventilation systems, mixing is due to mechanical dispersion and molecular diffusion. The proposed linear response model is applicable to systems where molecular diffusion is negligible. Accordingly, we define the mixing efficiency $\mu$ to describe the extent of mixing resulting from factors other than molecular diffusion:

$\mu= \begin{cases}1 & \text { for complete mixing } \\ 0 & \text { for piston flow }\end{cases}$

and incomplete mixing is characterized by $0<\mu<1$.

There are two premises which must be met before the linear response model can be applied. The capable model accounting for the effects of molecular diffusion is only the dispersive model. The proposed model assumes that molecular diffusion is negligible, i.e., the RTD function of the tracer or existing gaseous contaminant and air are identical and both are related to the distribution of air velocities. Furthermore, the application of the model requires that an ideal tracer be injected into and measured in the flux concentration. The proposed linear response model thus assumes that injected and measured in the flux has the same response function as the tracer material. These conditions, however, are met which are restrictive for two reasons: (1) molecular diffusion is negligible compared with mechanical dispersion in most field situations, especially in large-scale systems, and (2) flux concentration samples are easy to obtain from typical occupied airspace.

The general ventilation systems are very complex. The complexities arise from field-scale heterogeneity that create significant variability in the air velocity. The complexities make characterization of mixing in ventilation system a very difficult task. The task becomes even more difficult when the systems are viewed as distributed-parameter systems. An alternative approach for considering spatially distributed parameter or processes is to quantify the lumped response of mixing in the system. The concept of mixing efficiency, therefore, is adopted for the purpose of this study.

The major difference between linear response model and dispersive model, therefore, is that the linear response model employs the concept of mixing efficiency, whereas the dispersive model utilizes the dispersivity/diffusivity that may be scale dependent. Therefore, the proposed model simulates incomplete free from the dispersivity/diffusivity limitation.

As an example of changing the mixing efficiency, consider now the complete-incomplete mixing pattern. If $\mu$ is changed from $0<\mu<1$ to 0 , then the complete-incomplete mixing will give the complete-piston flow; if $\mu$ is changed to 1 , then the resulting mixing pattern will be the complete 
mixing. As an example of changing the mixing type, again consider the complete-incomplete mixing type. Suppose the mixing type is changed from complete-incomplete mixing to just complete mixing, the complete-incomplete mixing will give the complete mixing pattern. Cases 4 and 5, that are shown in Table 1, are more likely to occur in actual ventilation systems than piston flow, complete mixing, or the combination of complete-piston flow; and case 6 can be modeled differently by the dispersive model.

Analytical determination of the mean age in case of constant tracer input requires no additional information if the selected model is an one-parameter gamma pdf, such as piston flow or complete mixing patterns. For two-parameter gamma pdf, a priori knowledge of the parameters other than the mean age, the unknown, is necessary. Practically, however, a priori knowledge of these parameters is very difficult to obtain.

\section{Results and discussion}

\subsection{Numerical experiments}

Knowledge of the relative concentration $(U)$ and the air exchange rate $(\lambda)$, and the other parameters in Eq. (13), allows the determination of mean age of air $T$. The characteristics of the mixing behavior can be illustrated in Fig. 2, where $U$ is plotted against relative mean residence time $\lambda T$ for different mixing types for a constant input concentration and constant $\theta$ and $\eta$ with different $\mu$ of 0.25 and 0.75 , respectively. Fig. 2A shows that, for instance, for $U=0.8$ and $\mu=0.25$ the mean age is $T=0.4 / \lambda$ for complete-incomplete mixing pattern, whereas in incomplete mixing flow, mean age is $T=1 / \lambda$. The real age lies somewhere between the extreme values of Fig. 2. Its better estimate depends on the choice of an adequate mixing pattern. The relationship for the incomplete mixing reduces to those of the complete mixing for $\mu=1$ and $\alpha=1$, and the piston flow for $\mu=0$ and $\alpha=1$.

The RTD functions can assume any shape, depending on the values of the shape parameter $\alpha$. It is obvious that the flexibility of the RTD functions is very important if a realistic mixing type is derived to describe the complex mixing behavior of the general ventilation systems.

The minimum number of fitting parameters requires modeling the combinations of complete-incomplete or incomplete-piston flow is three: the mean age of air $(T)$, the mixing efficiency $(\mu)$, and mixing volume factor $\mathrm{I}(\theta)$ in the systems. On the other hand, the minimum number of parameters required to model the complete-incomplete-piston flow type is four: $T, \mu, \theta$, and mixing volume factor II, $\eta$.

Figs. 3A and B illustrate the RTD function of different shapes constructed for two of the mixing patterns developed in the study: the complete-incomplete mixing and the incomplete-piston flow types. Fig. 3A shows that the graph with $V_{\mathrm{c}}=0.9 V_{\mathrm{T}}(90 \%$ of the system is completely mixed),
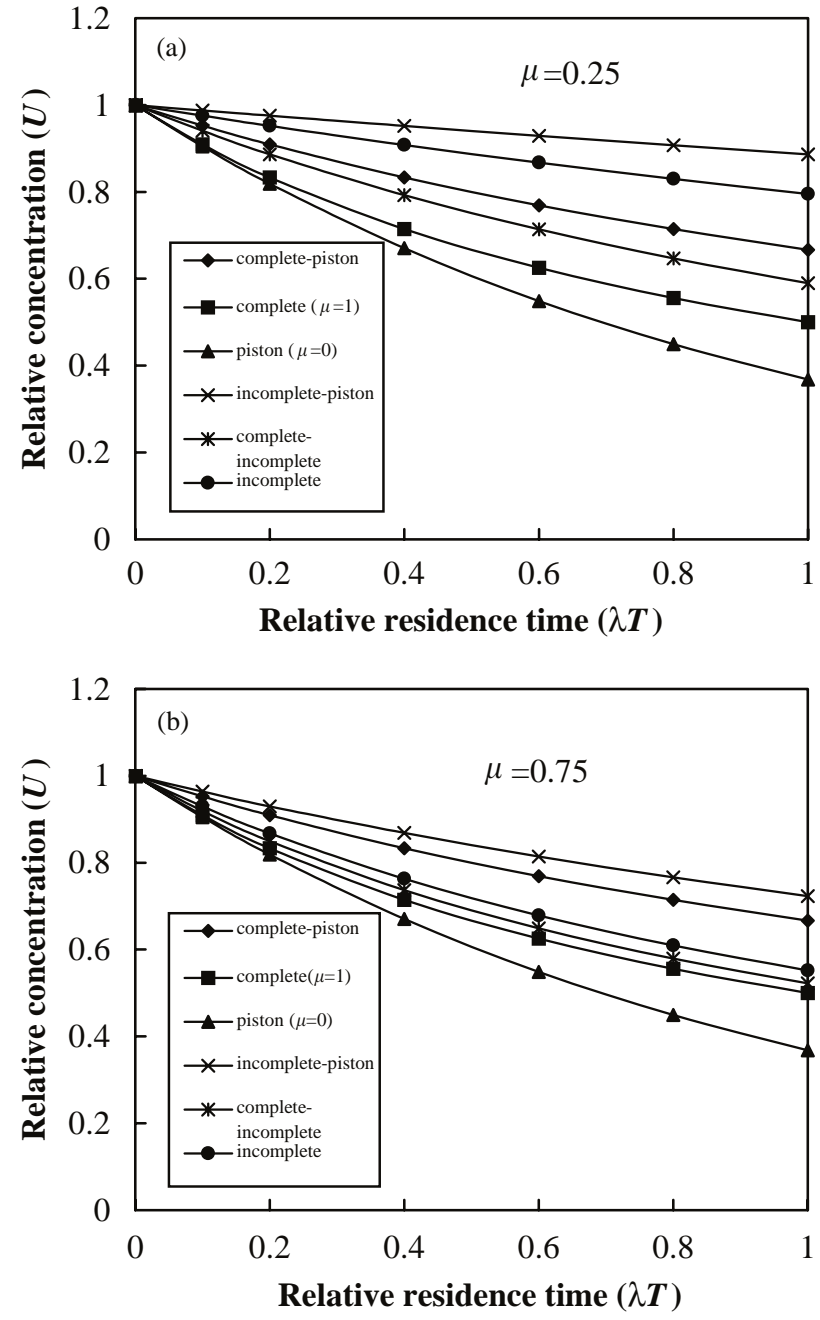

Fig. 2. Relative concentration as a function of the relative residence time for a constant input concentration and for different mixing models: (A) $\mu=0.25$ and (B) $\mu=0.75$.

closely approximates that of the complete mixing. Fig. 3B shows that as the volume occupied by piston flow increases, the variances of the graph decrease and their peaks increase, i.e., the graphs approach piston flow resulting from increasing $V_{\mathrm{p}}$ and decreasing $\mu$ imply piston flow. The numerical example reveals that the development of the completeincomplete-piston flow patterns with one set of parameters to account for the three mixing types (complete, incomplete, and piston flow) and their combinations.

\subsection{Fitting linear response model to chamber experiment}

Systems that tend to be highly mixed can be modeled with the incomplete mixing type with high mixing efficiency (i.e., the modeler has to specify a high value for $\mu$ ), or the complete-incomplete mixing pattern with high $\mu$ and/or high $V_{\mathrm{c}}$, or the complete-incomplete-piston flow with high $\mu$ and/or high $V_{\mathrm{c}}$. 

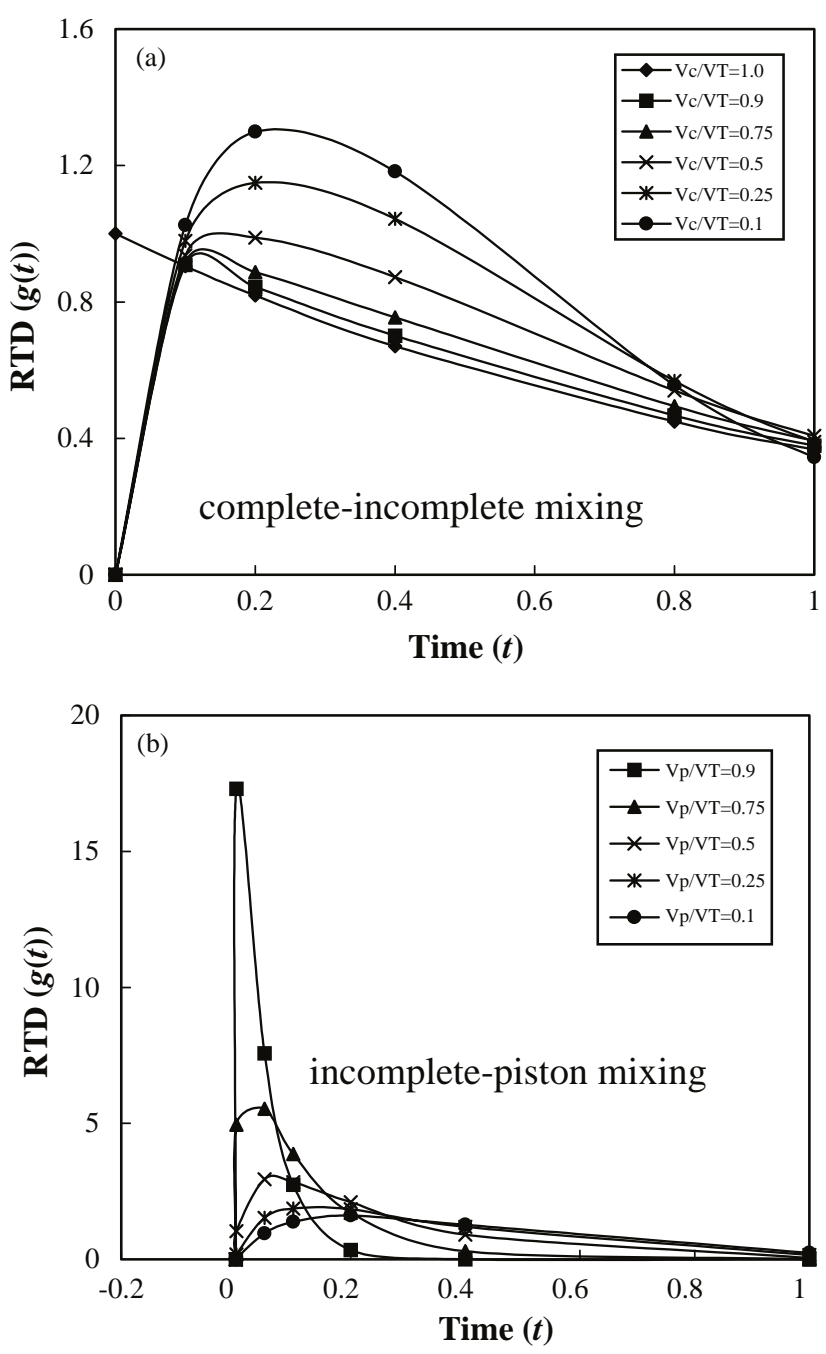

Fig. 3. The RTD function of different shapes constructed for two of mixing models: (A) complete-incomplete mixing and (B) incomplete-piston flow. $\left(V_{\mathrm{T}}=\right.$ total air volume, $V_{\mathrm{C}}=$ air volume occupied by complete mixing, $V_{\mathrm{P}}=$ air volume occupied by piston flow).

Systems characterized by a low degree of mixing, can be simulated by the incomplete mixing pattern with a low $\mu$, or the incomplete-piston flow with low $\mu$ and/or high $V_{\mathrm{p}}$ with low $\mu$ and/or high $V_{\mathrm{p}}$, or the complete-incomplete-piston flow with low $\mu$ and/or high $V_{\mathrm{p}}$. In the case of the completeincomplete-piston flow, the incomplete mixing volume $\left(V_{\mathrm{i}}\right)$ will provide a realistic transitional mixing zone between the highly or complete mixing volume $\left(V_{\mathrm{c}}\right)$ and the piston flow volume $\left(V_{\mathrm{p}}\right)$.

Four selected scenarios designated as Systems I, II, III, and IV that obtained from environmental chamber experiments were given to evaluate the applicability of the proposed linear response model. In our analysis, we eliminate the background carbon dioxide concentration $\left(C_{\mathrm{B}}\right)$ to reflect the characteristics of the output carbon dioxide dynamics. To select the proper RTDs for interpreting the transient times, we use the knowledge of the carbon dioxide output

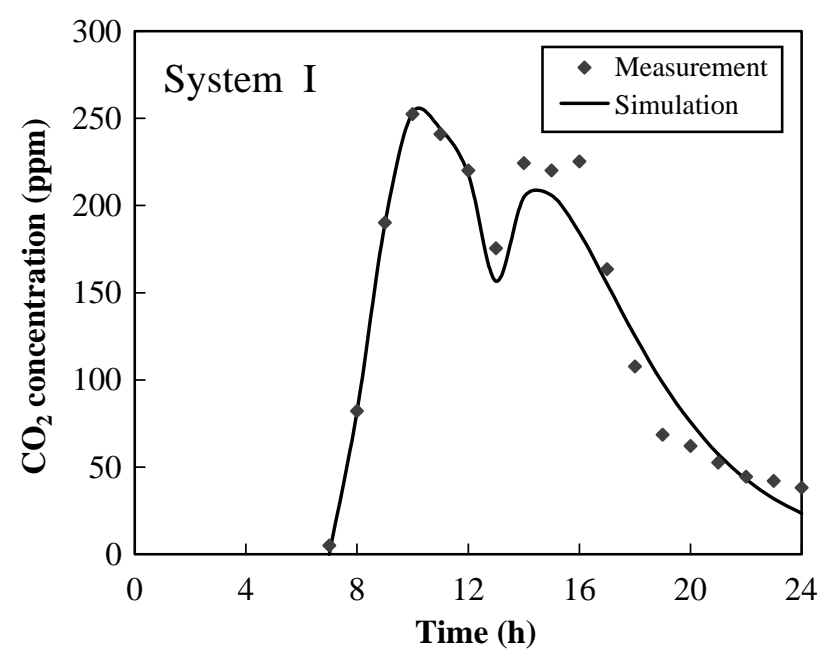

Fig. 4. Fitting of linear response model to System I as calculated from the complete-incomplete-piston mixing pattern with $\mu=0.34 \pm 0.18$ and $T=6.34 \pm 3.45 \mathrm{~h}$ (background $\mathrm{CO}_{2}$ concentration $=315 \mathrm{ppm}$ ).

concentrations as well as the nature of ventilation systems. Table 2 summarizes the simulation results of fitting model to the chamber experiments.

The fitting procedure in this work is preformed by guesswork and no objective criteria are applied. A low number of experimental data as well as a limited significance of the residence time of the investigated systems did not justify a great effort needed for a more rigorous reinterpretation. Whenever the fitting is performed by trial and error, the term "quantitative" is rather an arbitrary one.

Based on the above information, we chose the incomplete mixing pattern with relatively short $T$ and moderate $\mu$ for the interpretation of System I. Using this mixing pattern, the best fit is obtained for $T=6.34 \pm 3.45 \mathrm{~h}, \mu=0.34 \pm 0.18$. The model performance was evaluated by the root-mean-squared error (RMSE), computed from

$\mathrm{RMSE}=\sqrt{\frac{\sum_{n=1}^{N}\left[C_{\mathrm{m}, n}-C_{\mathrm{s}, n}\right]^{2}}{N}}$,

where $N$ denotes the number of measurements, $C_{\mathrm{m}, n}$ is the measurement data, and $C_{\mathrm{s}, n}$ is the simulation result corresponding to data point $n$. Table 2 lists the RMSE values for the model performances, indicating that RMSE values ranged from 2.10 to $15.70 \mathrm{ppm}$. The overall RMSE value of $8.64 \pm 5.25 \mathrm{ppm}$ is low, considering that the average standard error of measurements is already $11.07 \mathrm{ppm}$.

The predicted and observed output carbon dioxide concentration profiles for System I are shown in Fig. 4 in that the steady state is never obtained. This nonattainment of equilibrium leads to a large over estimate of the ventilation rate occurring in the test chamber. On comparison with the model, the RMSE value in the estimates as defined by Eq. (14) was $15.74 \mathrm{ppm}$, indicating that the numerics of the model were performing unfavorably well in the 13:0018:00 h shown in Fig. 4. The relatively large scatter of the 
Table 2

Simulation results of fitting the linear response model to measurements obtained from environmental chamber experiment

\begin{tabular}{|c|c|c|c|c|}
\hline System & $\mathrm{I}$ & II & III & IV \\
\hline $\begin{array}{l}\text { Mean residence time: } T \\
(\text { mean } \pm \mathrm{sd})(\mathrm{h})\end{array}$ & $6.43 \pm 3.45$ & $2.44 \pm 0.37$ & $2.80 \pm 0.22$ & $3.50 \pm 0.20$ \\
\hline $\begin{array}{l}\text { Mixing efficiency: } \mu \\
(m e a n \pm s d)\end{array}$ & $0.34 \pm 0.18$ & $0.53 \pm 0.02$ & $0.48 \pm 0.05$ & $0.56 \pm 0.01$ \\
\hline RMSE (ppm) & 15.70 & 2.10 & 5.43 & 11.34 \\
\hline Mixing pattern & Complete-incomplete-piston & Complete-incomplete & Complete-piston & Incomplete \\
\hline
\end{tabular}

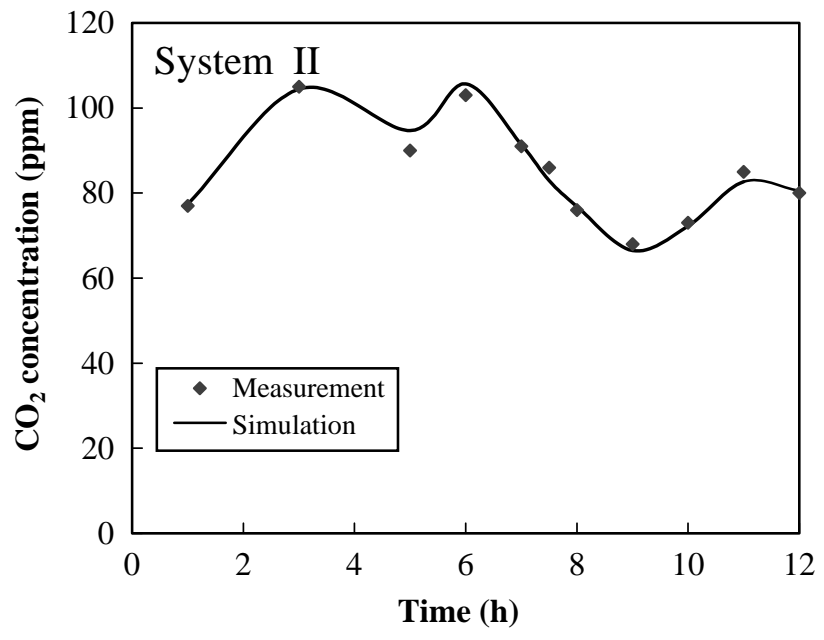

Fig. 5. Fitting of linear response model to System II as calculated from the complete-incomplete mixing pattern with $\mu=0.53 \pm 0.02$ and $T=2.44 \pm 0.37 \mathrm{~h}$ (background $\mathrm{CO}_{2}$ concentration $=315 \mathrm{ppm}$ ).

measurement data in 13:00-18:00 $\mathrm{h}$ was probably caused by the variable airflow rates. Therefore, more data would be necessary to interpret properly the mean residence time of this case. The interpretation gives an approximation of the mean residence time and mixing efficiency.

For System II, the complete-incomplete flow pattern with relatively low $T$ and moderate $\mu$ was selected. The best fit is obtained for $T=2.44 \pm 0.37 \mathrm{~h}, \mu=0.53 \pm 0.02$, and it is given in Fig. 5 in that the RMSE value is $2.10 \mathrm{ppm}$, indicating that the model were performing well. The interpretation of the data in this case conforms the use of more refined model gives more confidence and additional details concerning the physical parameters of the investigated systems.

For System III, the mean transient time of air in the system with the complete-piston mixing type was selected owing to the general tendency of such system to be highly mixed. Fig. 6 shows the optimal fit obtained $T=2.80 \pm 0.22 \mathrm{~h}$, $\mu=0.48 \pm 0.05$. In this case, the obtained fit is found to be favorably good at the RMSE value $5.43 \mathrm{ppm}$.

For System IV, the mean transient time of air in the system with the incomplete mixing pattern was selected owning to the general tendency of such system to be highly mixed. Fig. 7 shows that the best fit obtained $T=3.50 \pm 0.2 \mathrm{~h}$, and $\mu=0.56 \pm 0.2$. The RMSE value is found to be $11.34 \mathrm{ppm}$,

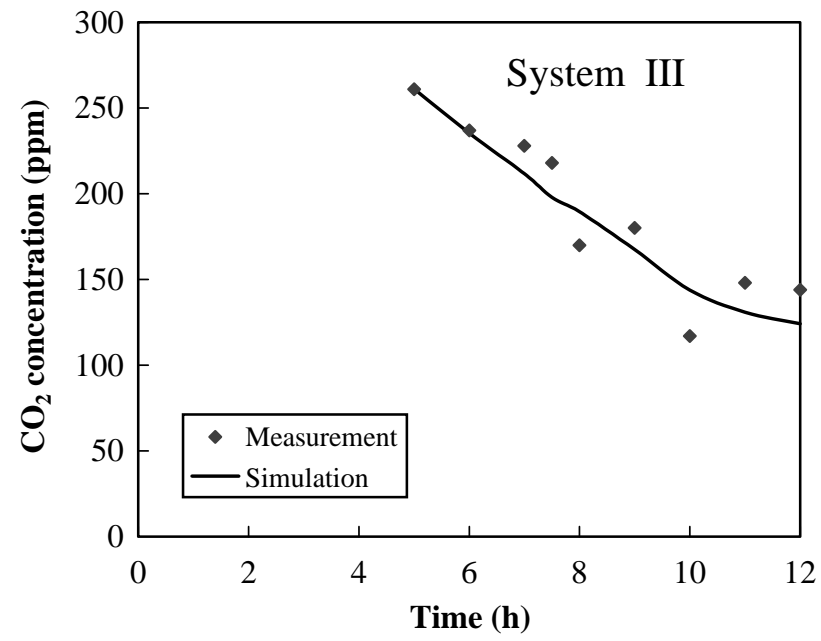

Fig. 6. Fitting of linear response model to System III as calculated from the complete-piston flow mixing with $\mu=0.48 \pm 0.05$ and $T=2.80 \pm 0.05 \mathrm{~h}$ (background $\mathrm{CO}_{2}$ concentration $=315 \mathrm{ppm}$ ).

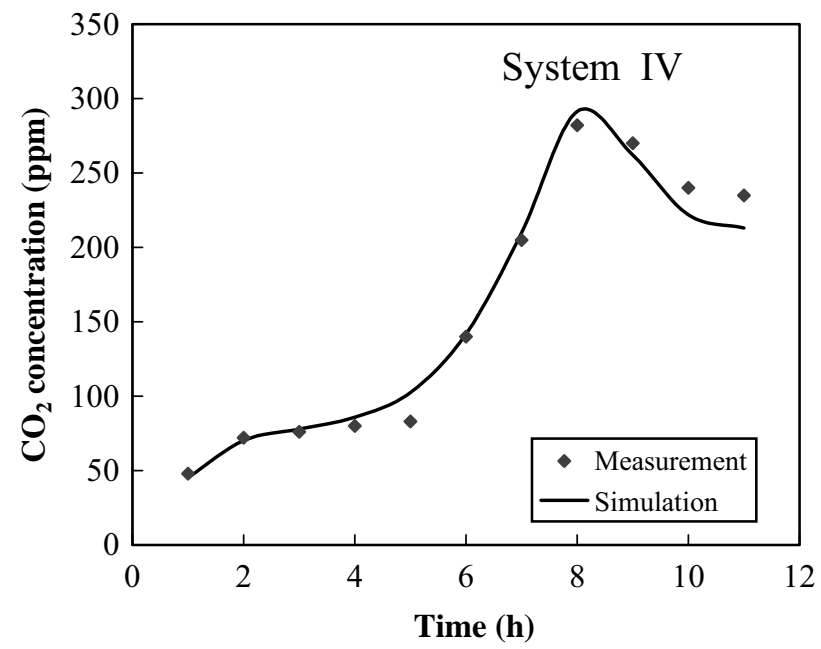

Fig. 7. Fitting of linear response model to System IV as calculated from the incomplete mixing pattern with $\mu=0.56 \pm 0.01$ and $T=3.50 \pm 0.20 \mathrm{~h}$ (background $\mathrm{CO}_{2}$ concentration $=315 \mathrm{ppm}$ ).

indicating that the deviation is well within the normal experimental accuracy.

The results of the present study illustrate a high degree of modeling success associated with the linear 
approximations, based exclusively on the measurements from the environmental chamber experiments. Simulation results also confirm that the two-parameter gamma RTD function gives a good fit and would be considered as more reliable in those cases. The results presented here should be interpreted as providing evidence that a linear approximation, invoking the principle of superposition, may be reasonable if the system parameters do not result in a dramatic shift in ventilation functions.

The linear response model supplies additional physical information on the ventilation system of $T$ or $\mu$. The linear response model does not require any physical knowledge prior to the data interpretation, but the obtained system response function supply physical information.

The results of the present study are generally supportive of the linear systems approach; a more rigorous test of the methodology would include application of dispersive model to an even broader consideration. Parsimony, however, is a legitimate modeling objective and additional model complexity (often in the form of nonlinearity) should only be advocated if it can be shown to significantly reduce uncertainties normally attributed to random system or measurement errors.

\section{Conclusions}

A simple linear response function model that is capable of interpreting output concentration profiles and simulating a variety of mixing regimes was presented. The gamma distribution was chosen because it is characterized by only two parameters. The mixing types of piston flow (no mixing), incomplete mixing, and complete mixing can be obtained from various forms of RTD functions. Incomplete mixing can be modeled separately or in combination with complete mixing or piston flow resulted in complete-incomplete and the incomplete-piston flow type, respectively.

Heterogeneous mixing is simulating free from the dispersivity limitations (scale dependence). The shape flexibility of the system response functions presented in this paper is comparable with those of the dispersive model. The linear response model has the potential for estimating mean residence time in ventilation systems characterized by different mixing regimes. In the case of the linear response model it is hoped that additional experimental data with well-documented test information can be used to confirm the overall applicability of a comparable linear approach to modeling indoor air quality responses to input disturbance.

\section{Appendix A. The deviation of $\alpha$ and $\beta$ in Eqs. (8) and (9)}

We consider a general case where the air volume relations in a general mixing pattern of a ventilated airspace in a time-invariant system is given in Eq. (7) as $V_{\mathrm{T}}=V_{\mathrm{c}}+V_{\mathrm{p}}+V_{\mathrm{i}}$. Apply this concept to Eq. (6) with respect to

$\alpha \beta=\frac{T}{\eta^{\prime}}$,

where $\eta^{\prime}=V_{\mathrm{T}} /\left(\mu\left(V_{\mathrm{c}}+V_{\mathrm{p}}+V_{\mathrm{i}}\right)\right)$.

The scalar parameter $\beta$ thus can be derived based on Eq. (A.1) and knowledge that for $V_{\mathrm{c}}: \mu=1$ and for $V_{\mathrm{p}}: \mu=0$,

$$
\begin{aligned}
\beta & =\frac{T}{\alpha \eta^{\prime}}=\frac{T}{\alpha\left[V_{\mathrm{T}} / \mu\left(V_{\mathrm{c}}+V_{\mathrm{p}}+V_{\mathrm{i}}\right)\right]}=\frac{T}{\alpha\left[V_{\mathrm{T}} /\left(V_{\mathrm{c}}+\mu V_{\mathrm{i}}\right)\right]} \\
& =\frac{T}{\alpha}\left[\frac{V_{\mathrm{c}}+\mu V_{\mathrm{i}}}{V_{\mathrm{T}}}\right] \\
& =\frac{T}{\alpha}\left[\frac{V_{\mathrm{c}}+\mu V_{\mathrm{i}}+\mu V_{\mathrm{p}}+\mu V_{\mathrm{c}}-\mu V_{\mathrm{c}}-\mu V_{\mathrm{p}}}{V_{\mathrm{T}}}\right] \\
& =\frac{T}{\alpha}\left[\frac{V_{\mathrm{c}}+\mu\left(V_{\mathrm{i}}+V_{\mathrm{p}}+V_{\mathrm{c}}\right)-\mu\left(V_{\mathrm{c}}+V_{\mathrm{p}}\right)}{V_{\mathrm{T}}}\right] \\
& =\frac{T}{\alpha}\left[\frac{V_{\mathrm{c}}}{V_{\mathrm{T}}}+\mu-\mu\left(\frac{V_{\mathrm{c}}+V_{\mathrm{p}}}{V_{\mathrm{T}}}\right)\right] .
\end{aligned}
$$

In view of Eq. (A.2), we define

$\theta=\frac{V_{\mathrm{T}}}{V_{\mathrm{c}}+V_{\mathrm{p}}}$

and make an appropriate transformation of $V_{\mathrm{T}} / V_{\mathrm{c}}$ to following expression as

$\frac{V_{\mathrm{T}}}{V_{\mathrm{c}}}=\frac{V_{\mathrm{T}}}{V_{\mathrm{c}}+V_{\mathrm{p}}} \frac{V_{\mathrm{c}}+V_{\mathrm{p}}}{V_{\mathrm{c}}}=\theta \eta$,

where $\eta=\left(V_{\mathrm{c}}+V_{\mathrm{p}}\right) / V_{\mathrm{c}}$ is already given in Eq. (6).

Substituting Eqs. (A.3) and (A.4) into Eq. (A.2), the final expression of scalar parameter $\beta$ can be obtained as

$\beta=\frac{T}{\alpha\left(V_{\mathrm{T}} /\left(V_{\mathrm{c}}+\mu V_{\mathrm{i}}\right)\right)}=\frac{T}{\alpha}\left(\frac{1}{\eta \theta}+\mu-\frac{\mu}{\theta}\right)$.

The shape parameter $\alpha$ can be derived as follows:

$$
\begin{aligned}
\alpha & =\frac{V_{\mathrm{T}}}{V_{\mathrm{c}}+V_{\mathrm{p}}+\mu V_{\mathrm{i}}}=\frac{V_{\mathrm{T}} / V_{\mathrm{T}}}{\left[\left(V_{\mathrm{i}}+V_{\mathrm{p}}+\mu V_{\mathrm{i}}\right) / V_{\mathrm{T}}\right]} \\
& =\frac{1}{\left[\left(V_{\mathrm{i}}+V_{\mathrm{p}}+\mu V_{\mathrm{i}}\right) / V_{\mathrm{T}}\right]} \\
& =\frac{1}{\left[\left(V_{\mathrm{c}}+V_{\mathrm{p}}\right) / V_{\mathrm{T}}+\mu V_{\mathrm{i}} / V_{\mathrm{T}}\right]} \\
& =\frac{1}{\left[\left(V_{\mathrm{c}}+V_{\mathrm{p}}\right) / V_{\mathrm{T}}+\mu\left(V_{\mathrm{i}}+V_{\mathrm{p}}+V_{\mathrm{c}}-V_{\mathrm{c}}-V_{\mathrm{p}}\right) / V_{\mathrm{T}}\right]} \\
& =\frac{1}{\left[\left(V_{\mathrm{c}}+V_{\mathrm{p}}\right) / V_{\mathrm{T}}+\mu\left(V_{\mathrm{i}}+V_{\mathrm{p}}+V_{\mathrm{c}}\right)-\mu\left(V_{\mathrm{c}}+V_{\mathrm{p}}\right) / V_{\mathrm{T}}\right]} \\
& =\frac{1}{\left[\left(V_{\mathrm{c}}+V_{\mathrm{p}}\right) / V_{\mathrm{T}}+\mu-\mu\left(\left(V_{\mathrm{c}}+V_{\mathrm{p}}\right) / V_{\mathrm{T}}\right)\right]} .
\end{aligned}
$$


Substituting Eq. (A.3) and definition of $\eta$ given in Eq. (6) into Eq. (A.6), we can obtain the final expression of shape parameter $\alpha$ as

$\alpha=\frac{1}{1 / \theta+\mu(1-1 / \theta)}$,

when $\mu \geqslant 0.5$.

\section{References}

[1] Barber EM, Ogilvie JR. Incomplete mixing in ventilated airspaces. Part I: Theoretical considerations. Canadian Agricultural Engineering 1982;24(1):25-9.

[2] Chen YC, Barber EM, Zhang Y, Besant RW, Sokhansanj S. Methods to measure dust production and deposition rates in buildings. Journal of Agricultural Engineering Research 1999;72:329-40.

[3] Levenspiel O. Chemical reaction engineering. New York: Wiley, 1999.

[4] Choi HL, Albright LD, Timmons MB, Warhaft Z. An application of the $k$-epsilon turbulence model to predict air distribution in a slot-ventilated enclosure. Transactions of the ASAE 1988;31(6):1804-14.

[5] Hoff SJ, Janni KA, Jacosbon LD. Three-dimensional buoyant turbulent flows in a scaled model, slot-ventilated, livestock confinement facility. Transactions of the ASAE 1992;35(2):671-86.

[6] Maghirang RG, Manbeck HB. Modeling particle transport in slot-inlet ventilated airspaces. Transactions of the ASAE 1993;36(5):1449-60.

[7] Liu Q, Hoff SJ, Maxwell GM, Bundy DS. Comparison of three k-epsilon turbulence models for predicting ventilation air jets. Transactions of the ASAE 1996;39(2):689-98.
[8] Soria M, Oliva A, Costa M, Perez-Segarra CD. Effect of contaminant properties and temperature gradients on the efficiency of transient gaseous contaminant removal from an enclosure: a numerical study. International Journal of Heat and Mass Transfer 1998;41:3589-609.

[9] Hoff SJ, Bundy DS. Comparison of contaminant dispersion modeling approaches for swine housing. Transactions of the ASAE 1996;39(3):1151-7.

[10] Nauman EB, Buffham BA. Mixing in continuous flow systems. New York: Wiley, 1983.

[11] Etheridge D, Sandberg M. Building ventilation: theory and measurement. New York: Wiley, 1996. p. 591-648.

[12] Freeman J, Gale R, Sandberg M. The efficiency of ventilation in a detached house. In: Energy efficient domestic ventilation systems for achieving acceptable indoor air quality. Proceedings of the Third AIC Conference, London, England, September 20-23, 1982. Bracknell, Berkshire, England: Air Infiltration Centre, 1982. p. $45-51$.

[13] Skarret E, Mathisen HM. International Symposium on Indoor Air Pollution, Health and Energy Conservation. MA: Amherst, 1981. p. 102-10.

[14] Grot RA, Lagus PL. Application of tracer gas analysis to industrial hygiene investigations. San Diego, CA: Lagus Applied Technology, Inc., 1991. p. 1-21.

[15] Buffham BA, Gibilaro LG. A generalization of the tanks-in-series mixing model. AIChE Journal 1968;14(5):805-6.

[16] Cholette A, Cloutier L. Mixing efficiency determinations for continuous flow systems. The Canadian Journal of Chemical Engineering 1959;37:105-12.

[17] Jennings BH, Armstrong JA. Ventilation theory and practice. ASHRAE Transactions 1971;77(I):50-60. 